\title{
芳杂环推拉型大分子的多光子上转换荧光性质
}

\author{
钱 鹰1,* 于文杰 ${ }^{1,2}$ 吕昌贵 ${ }^{2}$ 朱晓勤 ${ }^{1}$ 崔一平 2 \\ ( ${ }^{1}$ 东南大学化学化工学院, 南京 $211189 ;{ }^{2}$ 东南大学电子科学与工程学院, 南京 210096)
}

\begin{abstract}
摘要：用Wittig 反应和 Heck 反应制备了 2 个新的芳杂环推-拉型菼光大分子聚 [(2,5-二苯撑-1,3,4-啞二唑)$4,4^{\prime}$-乙烯撑-交替- $N, N^{\prime}$-二(4-苯乙烯撑)]苯胺 $\left(\mathrm{P}_{1}\right)$ 和聚 $[(2,5$-二苯撑- $1,3,4$-惡二唑)-4,4' -乙烯撑-交替- $N$-乙基-3,6-咔 唑乙烯撑 $]\left(\mathrm{P}_{2}\right) . \mathrm{P}_{1}$ 和 $\mathrm{P}_{2}$ 的分解温度分别为 373 和 $412{ }^{\circ} \mathrm{C}$, 热稳定性良好. 电化学性能用循环伏安法测定. $\mathrm{P}_{1}$ 和 $\mathrm{P}_{2}$ 的最高占有分子轨道 $(\mathrm{HOMO})$ 能级分别为 -5.39 和 $-5.81 \mathrm{eV}$, 最低未占有分子轨道(LUMO)能级分别为 -2.81 和 $3.09 \mathrm{eV}$. 用飞秒 Ti:Sapphire 激光器测定了 $\mathrm{P}_{1}$ 和 $\mathrm{P}_{2}$ 的三光子和双光子上转换苂光光谱. 在 $1250 \mathrm{~nm}$ 波长激发下, 在四氢呋喃溶液中 $\mathrm{P}_{1}$ 和 $\mathrm{P}_{2}$ 的三光子苂光发射峰分别位于 510 和 $491 \mathrm{~nm}$. 在 $800 \mathrm{~nm}$ 波长激发下, 在四氢呋喃溶 液中 $\mathrm{P}_{1}$ 和 $\mathrm{P}_{2}$ 的双光子菼光发射峰分别位于 511 和 $495 \mathrm{~nm}$. 在四氢呋喃溶液中大分子 $\mathrm{P}_{1}$ 和 $\mathrm{P}_{2}$ 单光子菼光发射 峰分别位于 503 和 $475 \mathrm{~nm}, \mathrm{P}_{1}$ 和 $\mathrm{P}_{2}$ 的荧光量子产率分别为 0.80 和 0.31 . 研究了多光子荧光发射过程的溶剂效 应. 结果表明, 溶剂极性增大, $\mathrm{P}_{1}$ 和 $\mathrm{P}_{2}$ 的多光子苂光发射波长明显红移.
\end{abstract}

关键词：芳杂环大分子; 上转换苂光; 三光子苂光; 双光子苂光; 溶剂效应 中图分类号: 0644

\section{Multiphoton Upconversion Fluorescence Properties of Heteroaromatic Push-Pull Polymer}

\author{
QIAN Ying, ${ }^{1, *} \quad$ YU Wen-Jie ${ }^{1,2} \quad$ LÜ Chang-Gui ${ }^{2} \quad$ ZHU Xiao-Qin ${ }^{1} \quad$ CUI Yi-Ping ${ }^{2}$ \\ ( ${ }^{1}$ School of Chemistry and Chemical Engineering, Southeast University, Nanjing 211189, P. R. China; \\ ${ }^{2}$ School of Electronic Science and Engineering, Southeast University, Nanjing 210096, P. R. China)
}

\begin{abstract}
Two novel heteroaromatic push-pull fluorescence polymers poly [(2,5-diphenylene-1,3,4-oxadiazole)-4,4'vinylene-alt- $N, N^{\prime}$-bis(4-phenylenevinylene)-phenylamine] $\left(\mathrm{P}_{1}\right)$ and poly [(2,5-diphenylene-1,3,4-oxadiazole)-4,4' -vinylenealt- $N$-ethyl-3,6-carbazolevinylene] $\left(\mathrm{P}_{2}\right)$ were synthesized by the Wittig reaction and the Heck reaction. Polymers $\mathrm{P}_{1}$ and $\mathrm{P}_{2}$ have decomposition temperatures of 373 and $412{ }^{\circ} \mathrm{C}$, respectively, and exhibit good thermal stabilities. Their electrochemical properties were explored by cyclic voltammetry. The highest occupied molecular orbital (HOMO) energy levels of $\mathrm{P}_{1}$ and $\mathrm{P}_{2}$ were -5.39 and $-5.81 \mathrm{eV}$, respectively, and the lowest unoccupied molecular orbital (LUMO) energy levels of $\mathrm{P}_{1}$ and $\mathrm{P}_{2}$ were -2.81 and $-3.09 \mathrm{eV}$, respectively. Three-photon and two-photon upconversion fluorescence spectra of both polymers were measured using a femtosecond Ti:sapphire that was pumped by a femtosecond laser at 1250 and $800 \mathrm{~nm}$. Strong three-photon upconversion fluorescence with maximum emission wavelengths of $510 \mathrm{~nm}$ for $\mathrm{P}_{1}$ and $491 \mathrm{~nm}$ for $\mathrm{P}_{2}$ in THF solution were observed. By pumping at $800 \mathrm{~nm}$, strong two-photon upconversion fluorescences at $511 \mathrm{~nm}$ for $\mathrm{P}_{1}$ and $495 \mathrm{~nm}$ for $\mathrm{P}_{2}$ in THF were measured. Maximum peaks of single-photon fluorescence in THF were at $503 \mathrm{~nm}$ for $\mathrm{P}_{1}$ and at $475 \mathrm{~nm}$ for $\mathrm{P}_{2}$ with fluorescence quantum yields of 0.80 for $\mathrm{P}_{1}$ and 0.31 for $\mathrm{P}_{2}$. The effect of solvent polarity on multi-photon fluorescence was studied. Increased solvent polarity were found to induce a marked red-shift of the emission band in polymers $\mathrm{P}_{1}$ and $\mathrm{P}_{2}$.
\end{abstract}

Key Words: Heteroaromatic polymer; Upconversion fluorescence; Three-photon fluorescence; Two-photon fluorescence; Solvatochromic effect

Received: October 10, 2008; Revised: March 6, 2009. Published on Web: April 3, 2009.

*Corresponding author. Email: yingqian@seu.edu.cn; Tel: +8625-84038250.

国家自然科学基金(60678042,60877024)和东南大学科学基金(9207041399)资助

C Editorial office of Acta Physico-Chimica Sinica 
分子同时吸收 3 个相同频率的光子被激发至高 能级, 经过弛豫过程后发生自发跃迁, 通过辐射形式 放出光子回到基态, 产生三光子上转换苂光现象. 三 光子苂光采用波长较长的、在生物组织中穿透能力 较强的红外激光作为激发光源, 可以避免普通苂光 成像中的苂光漂白和对生物细胞的光致毒问题, 且 生物组织对这一波段的线性吸收与 Rayleigh 散射 均比较小, 可以在深度三维空间的任意位点上引发 光物理过程和光化学过程, 大大提高了透射深度和 探测深度. 另外, 由于三光子过程与人射光强的三次 方成正比而具有更高的空间和时间分辨率和较高的 抑制噪声的能力, 图像对比度高, 可获得深层活组织 的影像, 使得在活细胞中研究复杂过程如 DNA 的 复制成为可能 ${ }^{[1-3]}$. 三光子泵浦荧光材料在三维高密 度信息存储、光动力学治疗、生物体三维成像、苂光 标记和结构识别探针、三光子上转换激光、短脉冲光 通讯等领域具有极为广阔的应用前景. 三光子荧光 对应于五阶非线性光学过程, 较难观测到, 影响因素 复杂, 关于有机分子尤其是聚合物大分子的三光子 苂光性质研究报道极少, 从理论和实验上寻找和设 计制备高效三光子苂光材料成为光电子领域的热点 研究课题之一 ${ }^{[4-6]}$.

三苯胺、咔唑、腎二唑均为双光子吸收的有效结 构单元 ${ }^{[7-13]}$, 本文把 2,5-二苯基噁二唑和三苯胺或 9乙基咔唑交替相连形成推-拉电子共轭结构, 制备了 2 个具有高效的双光子和三光子苂光性质的大分子. 用飞秒 Ti:Sapphire 锁模激光器分别在 3 倍线性吸 收波长 $1250 \mathrm{~nm}$ 和 2 倍线性吸收波长 $800 \mathrm{~nm}$ 激发 下, 研究这 2 个芳杂环大分子的三光子和双光子泵 浦上转换苂光性质及溶剂效应, 并与线性苂光性质 进行对比.

\section{1 实验部分}

\section{1 试剂、仪器及相关测试}

测量多光子苂光时所用激光器系统是来自于美 国 Coherent 公司的钛宝石飞秒激光系统, 包括飞秒 振荡器、放大器和 OPA 波长调谐系统( Mira $900 \mathrm{~F}$, Legend-F, OPerA). 光栅光谱仪置于垂直于通光方向 的一侧, 被激发的苂光经过约 $1 \mathrm{~mm}$ 的溶液后到达 光谱仪. 测量双光子苂光时直接使用从放大器输出 的波长为 $800 \mathrm{~nm}$ 、重复频率为 $1 \mathrm{kHz}$ 、脉宽小于 150 fs 的激光脉冲; 测量三光子苂光时使用经过 OPA (optical parametric amplifier) 波长调谐系统输出的
波长为 $1250 \mathrm{~nm}$ 、重复频率为 $1 \mathrm{kHz}$ 、脉宽约 $80 \mathrm{fs}$ 的 激光脉冲.

紫外-可见吸收光谱在日本 Shimadu UV-3600 型紫外-可见分光光度计上测得, 单光子苂光光谱用 英国 Edinburgh FLS-920 苂光光谱仪测定, 苂光量子 产率采用稀溶液比较法, 以硫酸奎宁为参比测定 $(\Phi=0.55)$. 溶剂均为国产分析纯, 使用前经回流脱水 处理.

循环伏安实验在上海辰华仪器有限公司生产的 CHI830型电化学工作站上进行, 测量时采用三电极 体系. 工作电极 (WE) 为石墨电极; 参比电极 $(\mathrm{RE})$ 为 饱和甘录电极 (SCE); 辅助电极 (CE) 为铂丝电极. 选 用的支持电解质是浓度为 $0.1 \mathrm{~mol} \cdot \mathrm{L}^{-1}$ 的四丁基高氯 酸胺(TBAP) 溶液, 溶剂为二氯甲烷(分析纯), 以二茂 铁为内标. 测试前向电解质溶液中通氮气以彻底除 氧, 整个实验过程处于氮气气氛, 测试样品浓度为 1 $\mathrm{g} \cdot \mathrm{mL}^{-1}$. 扫描范围: $+2--2 \mathrm{~V}$, 扫描速度为 $50 \mathrm{mV} \cdot \mathrm{s}^{-1}$.

大分子 $\mathrm{P}_{1}$ 和 $\mathrm{P}_{2}$ 的三光子泵浦频率上转换荧光 用 Ti:Sapphire 飞秒振荡器, 光学参量放大器系统 (model Mira 900-F, Legend-F, OperA, Coherent Inc.) 和 $O P A$ 测定. 大分子 $\mathrm{P}_{1}$ 和 $\mathrm{P}_{2}$ 的双光子葲浦苂光用 $\mathrm{Ti}$ : Sapphire 飞秒激光器, 光学参量放大器系统 (model Mira 900-F, Legend-F, OperA, Coherent Inc.) 和OMA (optical multichannel analyser)测定.

在 TA instruments SDT Q600 型热重分析仪测 试了化合物 $P_{1}$ 和 $P_{2}$ 的热失重行为, 热失重测试在 氮气环境下进行, 升温速率为 $20{ }^{\circ} \mathrm{C} \cdot \mathrm{min}^{-1}$.

\section{2 材料合成}

\subsubsection{2,5-二(4-埧甲基苯基)-1,3，4-嘿二唑的合成}

$250 \mathrm{~mL}$ 三口瓶中, 加人 $6 \mathrm{~g}(24 \mathrm{mmol}) 2,5$-二(4甲基苯基)-1,3,4-噁二唑, $9.4 \mathrm{~g}(52 \mathrm{mmol}) N$-溴代丁二 酰亚胺, $0.1 \mathrm{~g}$ 过氧化苯甲酰, 溶于 $120 \mathrm{~mL}$ 四氯化碳 中. 加热至 $80{ }^{\circ} \mathrm{C}$, 回流反应 $6 \mathrm{~h}$. 反应结束后趁热过 滤混合物, 滤饼用热水洗涤 $(3 \times 100 \mathrm{~mL})$, 干燥, 所得 固体用四氢呋喃(THF)/乙醇(体积比 1:1)混合溶剂重 结晶, 得到 2,5-二(4-溴甲基苯基)-1,3,4-噁二唑的白 色针状晶体, 产率: $65 \%$. 熔点 $226-227{ }^{\circ} \mathrm{C}, \mathrm{IR}(\mathrm{KBr}$ 压片) $\sigma / \mathrm{cm}^{-1}: 3050,1552,1494,1347,1072 ;{ }^{1} \mathrm{H} \mathrm{NMR}$ $\left(\mathrm{CDCl}_{3}\right) \delta: 4.55\left(\mathrm{~s}, 4 \mathrm{H}, 2\right.$ 组一 $\left.\mathrm{CH}_{2}-\right), 7.59(\mathrm{~d}, 4 \mathrm{H}, \mathrm{Ph}-$ $\mathrm{H}, J=7.95 \mathrm{~Hz}), 8.14(\mathrm{~d}, 4 \mathrm{H}, \mathrm{Ph}-\mathrm{H}, J=7.92 \mathrm{~Hz})$

1.2.2 2,5-二(4-乙烯基苯基)-1,3,4-嘿二唑的合成

$100 \mathrm{~mL}$ 三口瓶中, 加人 $8.2 \mathrm{~g}(2.69 \mathrm{mmol}) 2,5-二$ (4-溴甲基苯基)-1,3,4-噁二唑, $7.3 \mathrm{~g}$ (过量)三苯基磷 
和 $34 \mathrm{~mL}$ 苯. 加热升温至 $80{ }^{\circ} \mathrm{C}$, 磁力搅拌, 反应 12 h. 用乙酸乙酯/石油醚(体积比为 $1: 3$ ) 混合溶剂重结 晶, 得到 $12.9 \mathrm{~g}$ 白色针状晶体, 即为 1,3,4-噁二唑2,5-二苠基溴化三苯基镂, 产率 92.3\%。1,3,4-噁二 唑-2,5-二苄基溴化三苯基磷与甲醛在室温下缩合, 用乙醚萃取, 用柱色谱提纯, 得到 2,5-二(4-乙烯基苯 基)-1,3,4-噁二唑的 $\mathrm{IR}\left(\mathrm{KBr}\right.$ 压片)的 $\sigma\left(\mathrm{cm}^{-1}\right): 3050$, $1612,1548,1494,1070,844 ;{ }^{1} \mathrm{H} \mathrm{NMR}\left(\mathrm{CDCl}_{3}\right) \delta: 5.40$ (d, $\left.2 \mathrm{H}, J=10.89,=\mathrm{CH}_{2}\right), 5.87\left(\mathrm{~d}, 2 \mathrm{H}, J=17.58,=\mathrm{CH}_{2}\right)$, 6.74-6.83(m, $2 \mathrm{H},=\mathrm{CH}-)$, 7.59(d, $4 \mathrm{H}, J=7.38, \mathrm{Ph}-$ $\mathrm{H}), 8.32(\mathrm{~d}, 4 \mathrm{H}, J=8.28, \mathrm{Ph}-\mathrm{H})$

\subsection{3 大分子 $P_{1}$ 的合成}

在 $50 \mathrm{~mL}$ 三颈瓶中将双(4-甲酰基苯基)苯胺 $(0.30$ $\mathrm{g}, 1 \mathrm{mmol}$ )溶于 $N, N^{\prime}$-二甲基甲酰胺(DMF, $4 \mathrm{~mL}, 51.6$ $\mathrm{mmol}$ )中. 氮气保护, 磁力搅拌下滴加溶有 1,3,4-噁 二唑-2,5-二芐基溴化三苯基磷 $(0.93 \mathrm{~g}, 1 \mathrm{mmol})$ 的 4 $\mathrm{mL}$ DMF 溶液. 滴完后升温至 $35-40{ }^{\circ} \mathrm{C}$, 缓慢滴加溶 有叔丁醇钾 $(0.448 \mathrm{~g}, 4 \mathrm{mmol})$ 的 $6 \mathrm{~mL}$ 乙醇溶液. 滴 加完毕, 磁力搅拌下, 常温反应 $12 \mathrm{~h}$. 反应结束后, 将 反应液倒人乙醇中, 生成大量絮状沉淀, 抽滤, 用热 乙醇 $\left(60{ }^{\circ} \mathrm{C}\right)$ 洗涤 $(3 \times 50 \mathrm{~mL})$, 用二氯甲烷重结晶, 得 到橘红色粉末 $0.8 \mathrm{~g}$, 产率 $70 \%$. IR( $\mathrm{KBr}$ 压片)的 $\sigma\left(\mathrm{cm}^{-1}\right)$ : $3023,1589,1504,1415,1265,1176,1063,961 ;{ }^{1} \mathrm{H}$ NMR(300 MHz, $\left.\mathrm{CDCl}_{3}\right) \delta$ : 8.03-8.13 (m, $\left.\mathrm{Ph}-\mathrm{H}\right), 7.65-$ $7.71(\mathrm{~m}, \mathrm{Ph}-\mathrm{H}), 7.40-7.52(\mathrm{~m}, \mathrm{Ph}-\mathrm{H}), 7.32-7.37(\mathrm{~m}$, $\mathrm{Ph}-\mathrm{H}), 7.11-7.23(\mathrm{~m}, \mathrm{Ph}-\mathrm{H}), 6.55-6.64(\mathrm{~m},-\mathrm{CH}=$ CH一); GPC 分析: 重均分子量为 6980, 数均分子量 为 5691 , 分子量分散系数为 1.23 .

\subsection{4 大分子 $P_{2}$ 的合成}

将 3,6-二溴-9-乙基咔唑 $(0.177 \mathrm{~g}, 0.5 \mathrm{mmol})$, 1,3,4-惡二唑- 2,5 -二苯乙烯 $(0.137 \mathrm{~g}, 0.5 \mathrm{mmol})$, 醋 酸钯 $\mathrm{Pd}(\mathrm{OAc})_{2}(0.0066 \mathrm{~g}, 0.03 \mathrm{mmol})$, 三(邻-甲基苯 基)磷 $(0.019 \mathrm{~g}, 0.06 \mathrm{mmol})$ 加人耐压管式反应器中, 加人 $N, N^{\prime}$-二甲基甲酰胺与三乙胺的混合溶剂 $6 \mathrm{~mL}$ (体积比为 15:1), 反应体系用氮气反复洗涤多次, 磁 力搅拌, $110{ }^{\circ} \mathrm{C}$ 密闭体系氮气氛中反应 $36 \mathrm{~h}$. 反应结 束后, 反应液冷却至室温, 倒人 $50 \mathrm{~mL}$ 甲醇中, 搅拌, 形成粉末状沉淀, 抽滤, 用甲醇多次洗涤, 抽滤, 干 燥. 得黄绿色固体 $0.16 \mathrm{~g}$. 产率 $60 \%$. IR( $\mathrm{KBr}$ 压片)的 $\sigma\left(\mathrm{cm}^{-1}\right) 3030,2962,2926,1592,1491,1228,1179$, 1090, 962; ${ }^{1} \mathrm{H} \mathrm{NMR}\left(300 \mathrm{MHz}, \mathrm{CDCl}_{3}\right) \delta: 8.38-8.29$ $(\mathrm{m}, \mathrm{Ph}-\mathrm{H}), 8.15-8.07(\mathrm{~m}, \mathrm{Ph}-\mathrm{H}), 7.71-7.61(\mathrm{~m}, \mathrm{Ph}-$ $\mathrm{H}), 7.51-7.42(\mathrm{~m}, \mathrm{Ph}-\mathrm{H}), 6.92(\mathrm{~m},-\mathrm{CH}=\mathrm{CH}-)$,
$3.77-3.69\left(\mathrm{~m},-\mathrm{CH}_{2}-\right), 1.25\left(\mathrm{t}, J_{1}=6.99 \mathrm{~Hz}, J_{2}=7.05 \mathrm{~Hz}\right.$, $-\mathrm{CH}_{3}$ ); GPC 分析: 重均分子量为 2302 , 数均分子量 为 1794 , 分子量分散系数为 1.28 .

\section{2 结果与讨论}

\section{1 芳杂环大分子的制备方法及电化学性质和热 性能}

本文设计合成了 2 个芳杂环苂光大分子, 电子 传输型 2,5-二苯基噁二唑单元和空穴传输型三苯胺 $\left(\mathrm{P}_{1}\right)$ 或咔唑 $\left(\mathrm{P}_{2}\right)$ 通过共轭链交替相连形成推-拉电子 结构, 具有高效的双光子和三光子苂光性质, 具体结 构如图 1 .

将双(4-甲酰基苯基)苯胺与 1,3,4-噁二唑-2,5-二 芐基溴化三苯基鏻盐在叔丁醇钾碱催化下, 在 $N, N^{\prime}$ 二甲基甲酰胺溶液中发生 Wittig 聚合反应或 3,6-二 溴-9-乙基咔唑与 2,5-二(4-乙烯基苯基)-1,3,4-噁二 唑, 在醋酸钯和三-邻-甲基苯基磷催化下, 在 $N, N^{\prime}$ 二甲基甲酰胺与三乙胺的混合溶剂中、 $110{ }^{\circ} \mathrm{C}$ 密闭 体系氮气氛中发生 Heck 聚合反应制备三光子苂光 大分子 ${ }^{[14]}$. 分别采用 2 种方法制备共轭聚合物.

用热重分析测得大分子 $\mathrm{P}_{1}$ 和 $\mathrm{P}_{2}$ 的分解温度分 别为 373 和 $412{ }^{\circ} \mathrm{C}$. 图 $2(\mathrm{a})$ 为大分子 $\mathrm{P}_{1}$ 的热重分析 (TGA)曲线.

用循环伏安法测定了有机材料的能带结构 [15,16] 图 2(b)为大分子 $\mathrm{P}_{2}$ 的循环伏安曲线. 以二茂铁为

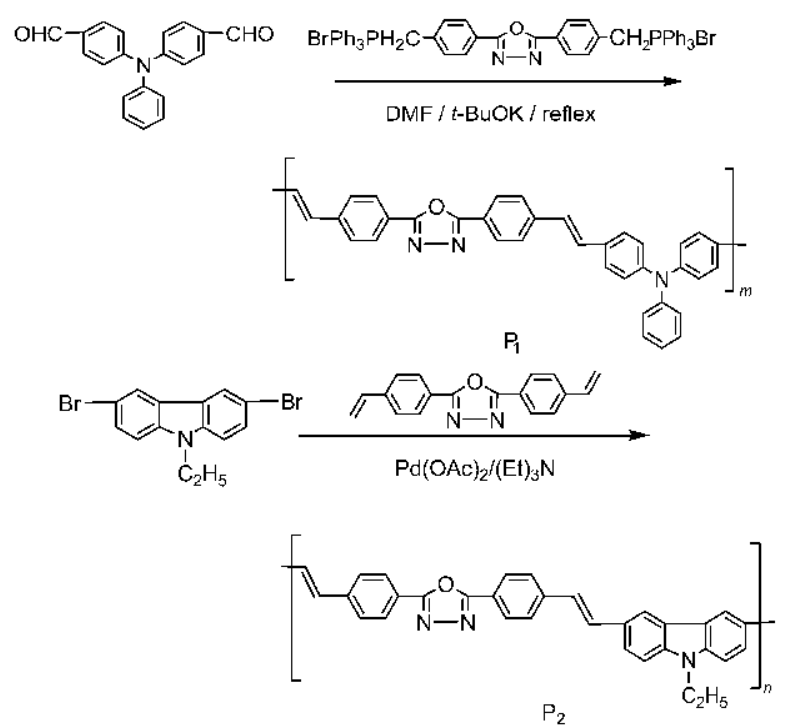

图 1 荧光大分子 $P_{1}$ 和 $P_{2}$ 的合成路线

Fig.1 Synthetic routes of polymers $P_{1}$ and $P_{2}$

$\mathrm{P}_{1}$ : poly[(2,5-diphenylene-1,3,4-oxadiazole)-4,4'-vinylene-alt- $N, N^{\prime}$-bis (4-phenylenevinylene)-phenylamine], $\mathrm{P}_{2}$ : poly[(2,5-diphenylene-1,3,4oxadiazole)-alt- $N$-ethyl-3,6-carbazolevinylene] 

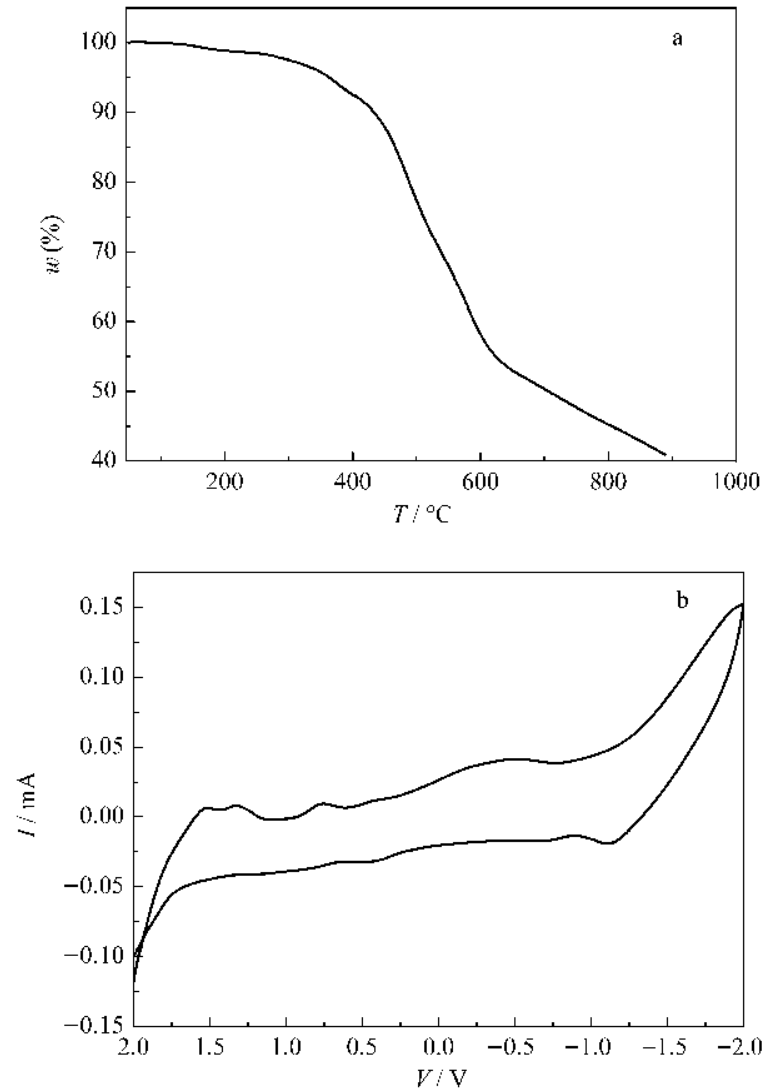

图 2 (a)大分子 $P_{1}$ 的 TGA 曲线, (b)大分子 $P_{2}$ 的 循环伏安曲线

Fig.2 (a) TGA thermogram of polymer $P_{1}$, (b) Cyclic voltammograms of polymer $\mathbf{P}_{2}$

内标, 测得 $\mathrm{P}_{1}$ 和 $\mathrm{P}_{2}$ 的 HOMO 能级分别为 -5.39 和 $-5.81 \mathrm{eV}$, 采用吸收光谱的边带法测得 $P_{1}$ 和 $P_{2}$ 的 LUMO 能级分别为 -2.81 和 $-3.09 \mathrm{eV}$.

\section{2 线性吸收和单光子荧光光谱}

芳杂环三光子荧光大分子 $\mathrm{P}_{1}$ 和 $\mathrm{P}_{2}$ 含有 2,5 -二 苯基噁二唑和三苯胺或 9-乙基咔唑基团, 其中空穴 传输型单元三苯胺、咔唑与电子传输型单元 2,5 -二 苯基噁二唑通过碳-碳双键交替相连, 形成具有推拉电子结构的共轭聚合物. 将聚合物 $\mathrm{P}_{1}$ 和 $\mathrm{P}_{2}$ 与它 们的小分子重复单元对比研究, 发现两者的吸收光 谱峰形非常相似. 图 3 为 $\mathrm{P}_{1}$ 和它的小分子重复单元 $\mathrm{m}_{1}$ 在相同质量分数二氯甲烷溶液中的紫外吸收光 谱, 均具有双吸收峰, 最大吸收波长分别在 417 和 $410 \mathrm{~nm}$, 聚合物与小分子相比共轭体系大为扩展, 分子跃迁能降低, 因而聚合物的吸收波长红移, 吸光 度也大为增加.

图 4 为苂光大分子的紫外吸收和苂光光谱. 大 分子 $P_{1}$ 和 $P_{2}$ 的紫外光谱都具有双峰结构, 在二氯

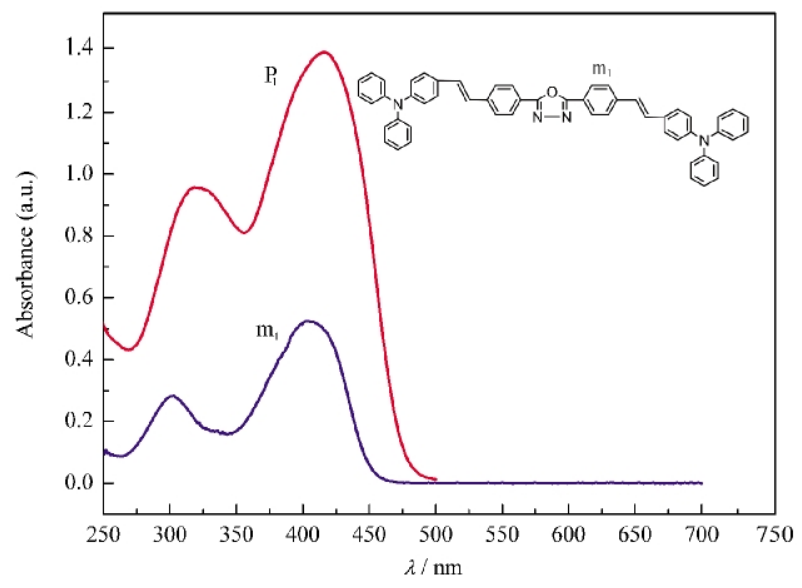

图 $3 P_{1}$ 和 $m_{1}$ 在二氯甲烷中的紫外吸收光谱

Fig.3 UV absorption spectra of $\mathrm{P}_{1}$ and $\mathrm{m}_{1}$ in $\mathrm{CH}_{2} \mathrm{Cl}_{2}$

甲烷溶液中分子内电荷转移态的共轭吸收分别在 417 和 $390 \mathrm{~nm}$. 与 $\mathrm{P}_{1}$ 相比, 噁二唑和 9-乙基咔唑的 交替共聚物 $\mathrm{P}_{2}$ 紫外吸收呈现明显蓝移. 三苯胺给电 子能力强于咔唑, 容易发生电荷转移, 基态与激发态 之间的能级差较低, 因而聚合物 $\mathrm{P}_{1}$ 的吸收光谱波长 较长.

在不同极性溶剂中大分子 $\mathrm{P}_{1}$ 的单光子苂光发
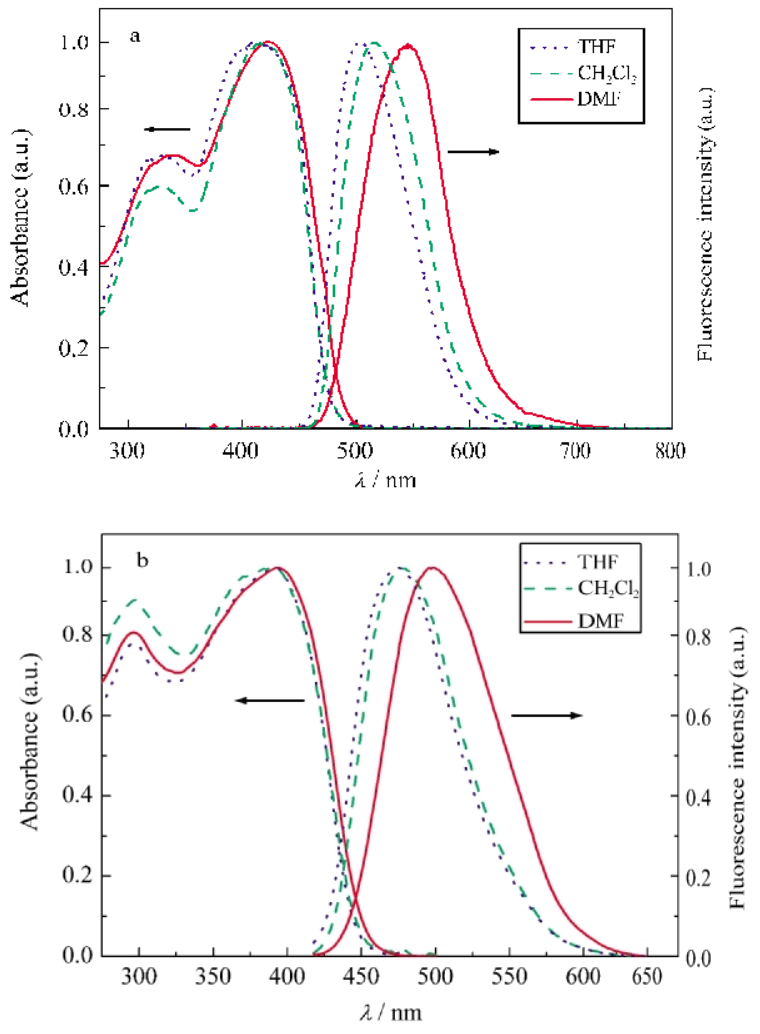

图 $4 \mathbf{P}_{1}(\mathrm{a})$ 和 $\mathbf{P}_{2}(\mathrm{~b})$ 在不同溶剂中归一化的紫外吸收和 荧光光谱

Fig.4 Normalized UV-absorption and fluorescence spectra of $P_{1}(a)$ and $P_{2}(b)$ in different solvents 
表 1 荧光大分子 $P_{1}$ 和 $P_{2}$ 的光物理性质

Table 1 Photophysical properties of fluorescence polymers $P_{1}$ and $P_{2}$

\begin{tabular}{llcccccc}
\hline & & $\lambda_{\text {max }}^{\text {abs }} / \mathrm{nm}$ & $\lambda_{\text {max }}^{\text {em }} / \mathrm{nm}$ & yield $(\Phi)$ & $\left(\nu_{\text {abs }}-\nu_{\mathrm{em}}\right) / \mathrm{cm}^{-1}$ & Two-photon fluorescence $(\mathrm{nm})^{\mathrm{a}}$ & ${\text { Three-photon fluorescence }(\mathrm{nm})^{\mathrm{b}}}^{\mathrm{b}}$ \\
\hline $\mathrm{P}_{1}$ & $\mathrm{THF}$ & $329 / 411$ & 503 & 0.80 & 4450 & 511 & 510 \\
& $\mathrm{CH}_{2} \mathrm{Cl}_{2}$ & $328 / 417$ & 515 & 0.10 & 4564 & 519 & 526 \\
& $\mathrm{DMF}$ & $340 / 421$ & 543 & 0.88 & 5337 & 565 & 566 \\
$\mathrm{P}_{2}$ & $\mathrm{THF}$ & $296 / 392$ & 475 & 0.31 & 4457 & 495 & 491 \\
& $\mathrm{CH}_{2} \mathrm{Cl}_{2}$ & $298 / 390$ & 476 & 0.08 & 4633 & 489 & 486 \\
& $\mathrm{DMF}$ & $296 / 394$ & 498 & 0.79 & 5301 & 516 & 513 \\
\hline
\end{tabular}

$\nu_{\mathrm{abs}}-\nu_{\mathrm{em}}:$ Stokes shift; a: $\lambda_{\mathrm{ex}}=800 \mathrm{~nm} ; \mathrm{b}: \lambda_{\mathrm{ex}}=1250 \mathrm{~nm}$
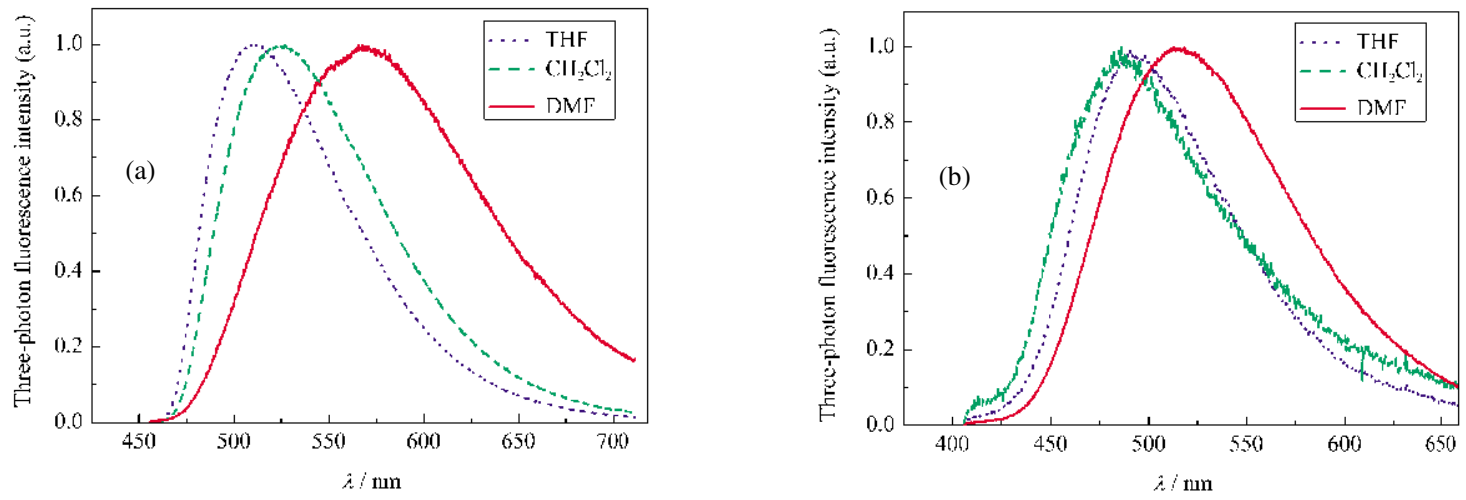

图 $5 P_{1}(a)$ 和 $P_{2}(b)$ 在不同溶剂中归一化的三光子荧光光谱(激发波长 $1250 \mathbf{n m}$ )

Fig.5 Normalized three-photon fluorescence spectra of $P_{1}(a)$ and $P_{2}(b)$ excited by $1250 \mathrm{~nm}$ femtosecond laser pulses in different solvents

射波长分别为 $503 \mathrm{~nm}(\mathrm{THF}), 51 \mathrm{~nm}\left(\mathrm{CH}_{2} \mathrm{Cl}_{2}\right)$ 和 543 $\mathrm{nm}(\mathrm{DMF})$, 大分子 $\mathrm{P}_{2}$ 的单光子苂光发射波长分别为 $475 \mathrm{~nm}(\mathrm{THF}), 476 \mathrm{~nm}\left(\mathrm{CH}_{2} \mathrm{Cl}_{2}\right)$ 和 $498 \mathrm{~nm}(\mathrm{DMF}), 2$ 个 分子的荧光光谱所反映出的溶剂化趋势类似, 随着 溶剂极性的增加, 苂光光谱红移, 苂光强度增加. 2 个大分子明显的溶剂化作用是由于激发态分子的偶 极矩比基态偶极矩大, 溶剂对激发态分子的稳定化 作用较大, 从而溶剂极性越大, 基态与激发态之间的 能量差越小, 荧光光谱红移 ${ }^{[17,18]}$. 采用稀溶液比较 法, 以硫酸奎宁为参比, 测定了大分子 $\mathrm{P}_{1}$ 和 $\mathrm{P}_{2}$ 的荧 光量子产率. 在 THF 溶液中, $\mathrm{P}_{1}$ 和 $\mathrm{P}_{2}$ 的苂光量子产 率分别为 0.80 和 0.31 . 表 1 列出了菼光大分子的线 性吸收和多光子荧光数据.

\section{3 三光子和双光子泵浦上转换荧光光谱}

大分子 $\mathrm{P}_{1}$ 和 $\mathrm{P}_{2}$ 的三光子泵浦频率上转换苂光 用 Ti:Sapphire 飞秒振荡器, 光学参量放大器系统测 定 ${ }^{[19]}$. 该波长可协调飞秒激光系统产生 $80 \mathrm{fs}$ 脉冲宽 度, $0.6 \mathrm{mrad}$ 发散角, $1 \mathrm{kHz}$ 重复频率特征参数的超 短脉冲. 在 1205-1570 nm 波段, 在飞秒脉冲的洜浦 下均能观察到明亮的频率上转换苂光. 如图 5 所示, 用线性吸收 3 倍波长的 $1250 \mathrm{~nm}$ 激发, 大分子 $\mathrm{P}_{1}$ 在 不同极性溶剂中的三光子频率上转换绿色苂光发射
波长分别为 $510 \mathrm{~nm}$ (THF), $526 \mathrm{~nm}\left(\mathrm{CH}_{2} \mathrm{Cl}_{2}\right)$ 和 $566 \mathrm{~nm}$ (DMF), 比单光子苂光波长分别红移了 7-23 nm. 大 分子 $\mathrm{P}_{2}$ 在不同极性溶剂中的三光子泵浦苂光发射 波长分别为 $491 \mathrm{~nm}$ (THF), $486 \mathrm{~nm}\left(\mathrm{CH}_{2} \mathrm{Cl}_{2}\right)$ 和 $513 \mathrm{~nm}$ (DMF), 比单光子荧光波长分别红移了 10-16 nm. 这是高浓度溶液中苂光重吸收的结果. 图 6 所示多 光子泵浦上转换苂光的过程是同时吸收多个光子使 处于基态的分子跃迁到激发态高振动能级, 然后弛 豫到第一激发态的最低振动能级, 最后通过辐射形 式放出光子回到基态. 所以苂光峰对应的光子能量 表示的是激发态最低振动能级到基态最高振动能级
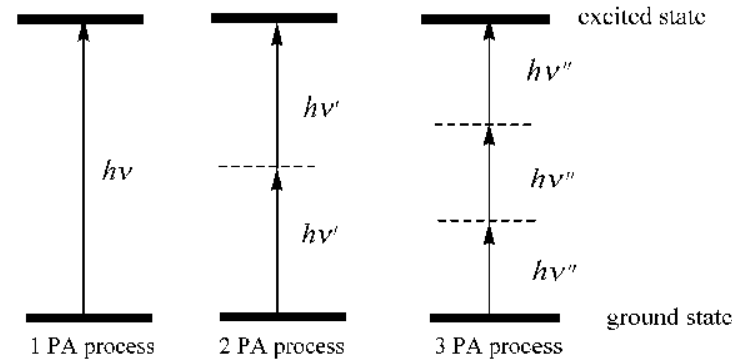

图 6 单光子和多光子诱导的分子跃迁过程

Fig.6 Elementary multiphoton-induced molecular transition process PA: photo absorption 

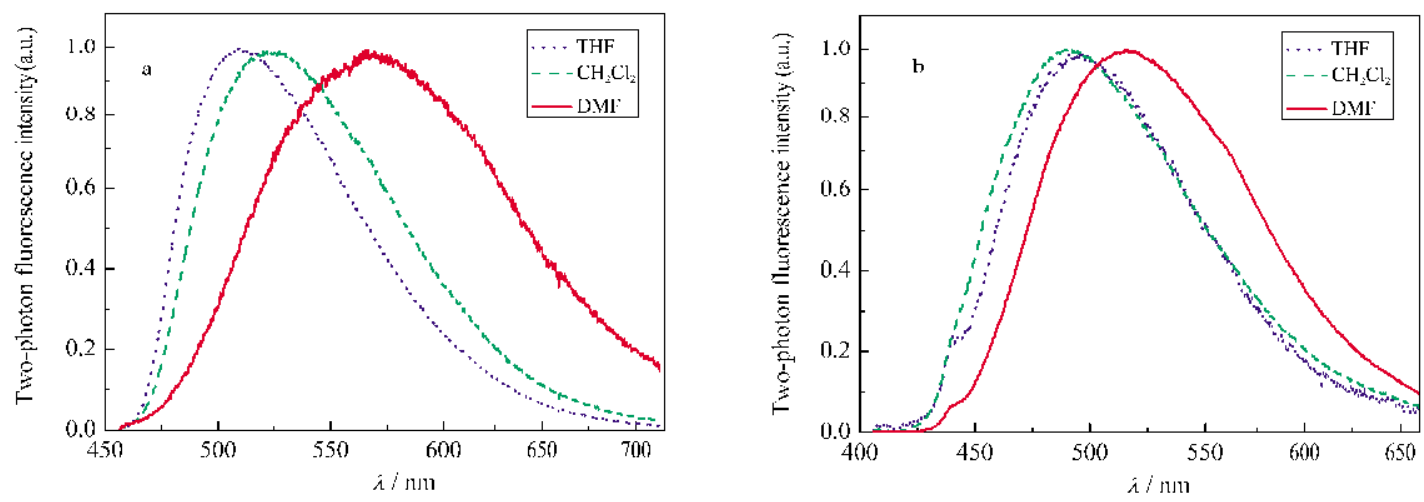

图 $7 \mathbf{P}_{1}(\mathrm{a})$ 和 $\mathbf{P}_{2}(\mathrm{~b})$ 在不同溶剂中归一化的双光子荧光光谱(激发波长 $800 \mathrm{~nm}$ )

Fig.7 Normalized two-photon fluorescence spectra of $P_{1}(a)$ and $P_{2}(b)$ excited by $800 \mathrm{~nm}$ femtosecond laser pulses in different solvents

的能量差. 单光子和多光子荧光的发射过程对应相 同的能级跃迁, 只是吸收过程不同, 因而溶剂对单光 子和多光子苂光的发射过程影响大致相同. 大分子 的三光子苂光也表现出了明显的溶剂效应, 随着溶 剂极性的增加 (从 $\mathrm{THF}$ 到 $\mathrm{CH}_{2} \mathrm{Cl}_{2}$ 到 DMF), 苂光发 射红移显著, $\mathrm{P}_{1}$ 和 $\mathrm{P}_{2}$ 在 $\mathrm{DMF}$ 溶液中的三光子荧光 发射峰比在 THF 溶液中分别红移了 56 和 $22 \mathrm{~nm}$.

大分子 $\mathrm{P}_{1}$ 和 $\mathrm{P}_{2}$ 的双光子泵浦荧光用Ti:Sapphire 飞秒激光器, 光学参量放大器系统和OMA测定 ${ }^{[20]}$. 测量双光子苂光时直接使用从放大器输出的波长为 $800 \mathrm{~nm}$ 、重复频率为 $1 \mathrm{kHz}$ 、脉宽小于 $150 \mathrm{fs}$ 的激光脉 冲. 如图 7 所示, 用线性吸收 2 倍波长的激光激发, 大分子 $\mathrm{P}_{1}$ 在不同极性溶剂中的双光子上转换绿色 苂光分别为 $511 \mathrm{~nm}$ (THF), $519 \mathrm{~nm}\left(\mathrm{CH}_{2} \mathrm{Cl}_{2}\right)$ 和 $565 \mathrm{~nm}$ (DMF), 大分子 $\mathrm{P}_{2}$ 的双光子苂光分别为 $495 \mathrm{~nm}$ (THF), $489 \mathrm{~nm}\left(\mathrm{CH}_{2} \mathrm{Cl}_{2}\right)$ 和 $516 \mathrm{~nm}$ (DMF). 2 个共轭大分子受 激光照射跃迁至激发态, 电荷由给体向受体转移, 分 子内各部分趋向平面, 形成发光的分子内电荷转移 态, 极性较大的溶剂有利于稳定分子内电荷转移态. 双光子苂光谱与三光子苂光谱非常相似, 溶剂效应 也一致. 由 2,5-二苯基啞二唑和三苯胺交替相连形 成的大分子 $\mathrm{P}_{1}$ 多光子菼光强度远大于由 2,5 -二苯基 噁二唑和9-乙基咔唑交替相连形成的大分子 $\mathrm{P}_{2}$.

\section{3 结 论}

分别采用双(4-甲酰基苯基)苯胺与 1,3,4-惡二 唑-2,5-二芐基溴化三苯基鏻盐在叔丁醇钾碱催化 下, 在 $N, N^{\prime}$-二甲基甲酰胺溶液中发生 Wittig 聚合 反应以及 3,6-二溴-9-乙基咔唑与 1,3,4-噁二唑-2,5二苯乙烯在醋酸钯和三-邻-甲基苯基磷催化下, 在
$N, N^{\prime}$-二甲基甲酰胺与三乙胺的混合溶剂中 $、 110{ }^{\circ} \mathrm{C}$ 密闭体系氮气氛中发生 Heck 聚合反应制备三光子 苂光大分子 $P_{1}$ 和 $P_{2} . P_{1}$ 和 $P_{2}$ 的分解温度分别为 373 和 $412{ }^{\circ} \mathrm{C}$, 热稳定性良好. 以二茂铁为内标, 用循环 伏安法测得 $\mathrm{P}_{1}$ 和 $\mathrm{P}_{2}$ 的 HOMO 能级分别为 -5.39 和 $-5.81 \mathrm{eV}$, 采用吸收光谱的边带法测得 $\mathrm{P}_{1}$ 和 $\mathrm{P}_{2}$ 的 LUMO 能级分别为 -2.81 和 $-3.09 \mathrm{eV}$.

研究飞秒 Ti:Sapphire 激光 1250 和 $800 \mathrm{~nm}$ 波 长激发下 2 个芳杂环推拉型大分子的三光子和双光 子泵浦上转换荧光性质及溶剂效应. 在四氢呋喃溶 液中大分子 $\mathrm{P}_{1}$ 和 $\mathrm{P}_{2}$ 和紫外吸收峰分别位于 411 和 $392 \mathrm{~nm}$, 单光子荧光发射峰分别位于 503 和 475 $\mathrm{nm}$, 双光子苂光发射峰分别位于 511 和 $495 \mathrm{~nm}$, 三 光子苂光发射峰分别位于 510 和 $491 \mathrm{~nm}$, 双光子苂 光谱与三光子苂光谱非常相似. 2 个共轭大分子受 激光照射跃迁至激发态, 电荷由给体向受体转移, 形 成发光的分子内电荷转移激发态, 极性较大的溶剂 有利于稳定激发态. 随着溶剂极性的增加, $\mathrm{P}_{1}$ 和 $\mathrm{P}_{2}$ 的多光子苂光发射峰发生红移. 单光子和多光子苂 光的发射过程对应相同的能级跃迁, 只是吸收过程 不同, 因而溶剂对单光子和多光子荧光的发射过程 影响大致相同. 由 2,5-二苯基噁二唑和三苯胺交替 相连形成的大分子 $\mathrm{P}_{1}$ 多光子苂光强度远大于由 2,5-二苯基嘒二唑和 9-乙基咔唑交替相连形成的大 分子 $\mathrm{P}_{2}$.

\section{References}

1 Tian, Y.; Chen, C. Y.; Yang, C. C.; Young, A. C.; Jang, S. H.; Chen, W. C.; Jen, A. K. Y. Chem. Mater., 2008, 20(5): 1977 2 Ha-Thi, M.; Penhoat, M.; Drouin, D.; Blanchard-Desce, M.; 
Michelet, V.; Leray, I. Chem .Eur. J., 2008, 14: 5941

3 Zheng, Q. D.; Xu, G. X.; Prasad, P. N. Chem. Eur. J., 2008, 14 5812

4 Samoc, M.; Morrall, J. P.; Dalton, G. T.; Cifuentes, M. P.; Humphrey, M. G. Angew. Chem. Int. Ed., 2007, 46(4): 731

5 Lin, N.; Ferrighi, L.; Zhao, X.; Ruud, K.; Rizzo, A.; Luo, Y. J. Phys. Chem. B, 2008, 112: 4703

6 He, G. S.; Tan, L. S.; Zheng, Q. D.; Prasad, P. N. Chem. Rev., 2008, 108(4): 1245

7 Tian, Y.; Chen, C. Y.; Cheng, Y. J.; Young, A. C.; Tucker, N. M.; Jen, A. K. Y. Adv. Funct. Mater., 2007, 17: 1691

8 Lartia, R.; Allain, C.; Bordeau, G.; Schmidt, F.; FioriniDebuisschert, C.; Charra, F.; Teulade-Fichou, M. P. J. Org. Chem., 2008, 73(5): 1732

9 Huang, P. H.; Shen, J. Y.; Pu, S. C.; Wen, Y. S; Lin, J. T.; Chou, P. T.; Yeh, M. C. J. Mater. Chem., 2006, 16(5): 850

10 Fitilis, I; Fakis, M.; Polyzos, I.; Giannetas, V.; Persephonis, P.; Vellis, P.; Mikroyannidis, J. Chem. Phys. Lett., 2007, 447: 300

11 Wei, P.; Bi, X. D.; Wu, Z.; Xu, Z. Org. Lett., 2005, 7(15): 3199

12 Chung, S. J.; Lin, T. C.; Kim, K. S.; He, G. S.; Swiatkiewicz, J.; Prasad, P. N.; Baker, G. A.; Bright, F. V. Chem. Mater., 2001, 13
(11): 4071

13 Belfield, K. D.; Bondar, M.; Hernandez, F. E.; Przhonska, O. V. J. Phys. Chem. C, 2008, 112(14): 5618

14 Jin, S. H.; Jung, J. E.; Park, D. K.; Jeon, B. C.; Kwon, S. K.; Kim, Y. H.; Moon, D. K.; Kim, S. H.; Gai, Y. S. Eur. Poly. J., 2001, 37: 921

15 Agrawal, A. K.; Jenekhe, S. A. Chem. Mater., 1996, 8(2): 579

16 Osaheni, J. A.; Jenekhe, S. A.; Perlstein, J. J. Phys. Chem., 1994, 98(48): 12727

17 Terenziani, F.; Droumaguet, C. L.; Katan, C.; Mongin, O.; Blanchard-Desce, M. ChemPhysChem, 2007, 8: 723

18 Katan, C.; Terenziani, F.; Mongin, O.; Wertz, M. H. V.; Porres, L.; Pons, T.; Mertz, J.; Tretiak, S.; Blanchard-Desce, M. J. Phys. Chem. A, 2005, 109(13): 3024

19 Lu, C. G.; Huang, W.; Luan, J. F.; Lu, Z. F.; Qian, Y.; Yun, B. F.; Hu, G. H.; Wang, Z. Y.; Cui, Y. P. Opt. Commun., 2008, 281(18): 4038

20 Signorini, R.; Ferrante, C.; Pedron, D.; Zerbetto, M.; Cecchetto, E.; Slaviero, M.; Fortunati, I.; Collini, E.; Bozio, R.; Abbotto, A.; Beverina, L.; Pagani, G. A. J. Phys. Chem. A, 2008, 112(18): 4224 